Nicolina Silvana Romano-Lieber 1

\title{
Revisão dos estudos de intervenção do farmacêutico no uso de medicamentos por pacientes idosos
}

Jorge Juarez Vieira Teixeira 1

Fatima Cristiane Lopes Goularte Farhat 2

Eliane Ribeiro 3

Márcia Terezinha Lonardoni Crozatti 4

Giane Sant'Ana Alves de Oliveira 2

1 Departamento de Prática de Saúde Pública,

Faculdade de Saúde Pública, Universidade de São Paulo.

Av. Dr. Arnaldo 715,

São Paulo, SP

01246-904, Brasil.

nicolina@usp.br

jorgetei@usp.br

2 Curso de Farmácia,

Faculdade de Ciências

da Saúde, Universidade

Metodista de Piracicaba.

Rodovia do Açúcar Km 156,

Piracicaba, SP

13400-911, Brasil.

bealagi@uol.com.br

farhat@terra.com.br

3 Hospital Universitário,

Universidade de São Paulo.

Av. Prof. Lineu Prestes 2565,

São Paulo, SP

05508-900, Brasil.

ribeiroromaro@zipmail.com.br

4 Departamento de

Farmácia e Farmacologia,

Universidade Estadual

de Maringá.

Av. Colombo 5790

Maringá, $P R$

87020-900, Brasil.

crozatti@maringa.com.br

\author{
A literature review on pharmacists' interventions \\ in the use of medication by elderly patients
}

\begin{abstract}
The total proportional geriatric population in Brazil is projected to increase from 9.05\% in 1999 to approximately 13\% in 2020. Non-communicable diseases are common in this age group, and medication is used frequently. Inadequate prescription and improper use of drugs can produce undesirable outcomes, leading to avoidable hospitalization and increasing health care costs. The objective of this paper was to conduct a literature review of pharmacists' interventions and their influence on use of medication by elderly patients, based on five databases from 1970 to 1999. The sample consisted of 76 studies, of which 15 were analyzed and discussed. Research on this subject is scarce, and limited to developed countries. In general, the interventions presented favorable outcomes. Most actions were limited to counseling patients and their physicians, and there was a lack of interventions to adjust the medication to the user.
\end{abstract}

Key words Aging Health; Pharmacists; Drug Utilization

Resumo No Brasil, os idosos representavam 9,05\% em 1999 e, em 2020, poderão totalizar 13\% da população. Nessa faixa etária as doenças crônicas e degenerativas são comuns e freqüentemente se utiliza muitos medicamentos. A prescrição e o uso inadequado dos mesmos podem levar a resultados indesejados, acarretando internações hospitalares evitáveis e elevando os custos do sistema de saúde. O objetivo deste trabalho foi conhecer os estudos de intervenção do farmacêutico e sua influência no uso de medicamentos pelo paciente idoso, listados em cinco bases de dados, no período entre 1970 e 1999. Foram localizados 76 artigos, resultando em 15 trabalhos para análise e discussão. Os estudos sobre o tema são escassos e limitados aos países de economia avançada. De uma forma geral, as intervenções apresentaram resultado positivo. A maioria das intervenções limitou-se ao aconselhamento ao usuário elou ao prescritor, notando-se falta de ações que levem à adequação do medicamento ao usuário.

Palavras-chave Saúde do Idoso; Farmacêuticos; Uso de Medicamentos 


\section{Introdução}

Embora o uso de medicamentos seja uma questão relevante em todas as faixas etárias, as pesquisas sobre o assunto têm se dedicado, com freqüência, ao paciente idoso, em decorrência das peculiaridades desse grupo etário.

No Brasil, dados de 1999 indicavam que o contigente de pessoas com 60 anos ou mais, era de 9,05\%, devendo alcançar 13\% em 2020 (IBGE, 1998, 1999). Nos Estados Unidos, as pessoas com 65 anos ou mais perfaziam 12,7\% da população no ano 2000, estimando-se que em 2030 serão $20 \%$ (DHHS, 2000). Uma das principais conseqüências dessa transformação demográfica se dá no financiamento do setor saúde, com uma participação desproporcional dos idosos na demanda por serviços, principalmente hospitalizações. Dados do Sistema Único de Saúde (SUS) mostram que essa faixa etária, em 1996, foi responsável por $23 \%$ dos custos hospitalares. A falta de serviços domiciliares e/ou ambulatoriais adequados faz, muitas vezes, com que o primeiro atendimento se dê em estágio avançado no hospital, aumentando os custos e diminuindo a possibilidade de um prognóstico favorável. Os problemas de saúde dos mais velhos, além de serem de longa duração, podendo prolongarse por 15 anos ou mais, requerem pessoal qualificado, equipe multidisciplinar, equipamentos e exames complementares, exigindo o máximo de recursos do sistema de saúde (MS, 1999).

As doenças crônicas e patologias degenerativas levam a uma maior demanda por medicamentos (Cartwright \& Smith, 1988; Landahl, 1987; Österlind \& Bucht, 1991). A maioria dos idosos utiliza mais de um medicamento periodicamente e quando hospitalizados recebem entre oito a quinze, representando um consumo de aproximadamente 30 a $35 \%$ de todos os medicamentos prescritos nos Estados Unidos (Baum et al., 1984; Ostrom et al., 1985; Piraino, 1995; Salom \& Davis, 1995). No Japão, em 1995, a população com 65 anos ou mais era de 14,5\%, com consumo de $30,6 \%$ de todos os medicamentos prescritos (Okuno et al., 1999). Já na Inglaterra, em 1985, os idosos receberam 39\% de todas as prescrições de medicamentos (Cartwright \& Smith, 1988).

A prescrição medicamentosa envolve o entendimento das mudanças próprias da idade, tanto estrutural quanto funcional, de vários órgãos e sistemas que podem alterar a farmacocinética e a farmacodinâmica de muitos medicamentos. Dessa forma, a prescrição inadequada para pacientes idosos aumenta o risco de reações adversas medicamentosas (RAMs) (Heller et al., 1984; Walker \& Wynne, 1994). Assim, em- bora os medicamentos contribuam de forma significativa para o tratamento de doenças prevalentes nos idosos, as reações adversas também ocorrem mais comumente e estão implicadas na ordem de 10 a $31 \%$ das admissões agudas em geriatria (Atkin \& Shenfield, 1995; Lamy, 1990; Nolan \& O’Malley, 1988). A não adesão ao tratamento que, segundo Morrow et al. (1988) e Kessler (1992), foi da ordem de 40 a 55\% nos Estados Unidos, nessa faixa etária, expõe o paciente a um maior risco de hospitalizações e morbidade.

Estudo realizado naquele país apontou que uma média de 5,3\% das admissões hospitalares, na população geral, estava relacionada ao não cumprimento da medicação prescrita, a um custo hospitalar direto, em 1986, de mais de US\$ 8 bilhões. Quando os custos indiretos foram incluídos, a estimativa total de gastos atingiu US\$ 25 bilhões (Sullivan et al., 1990).

Muitos fatores contribuem para diminuir o conhecimento do paciente idoso quanto ao seu tratamento medicamentoso. Isso inclui, entre outras causas, a falta de aconselhamento individualizado, a falta de informação escrita personalizada e reforço das instruções orais, inabilidade para recordar as informações previamente apresentadas e a falta de um ajudante ou auxiliar na hora de tomar a medicação (O'Connell \& Johnson, 1992). Nesse sentido, Gibbs et al. (1989) comentam que a despeito da vasta prescrição de medicamentos para os pacientes idosos, o conhecimento sobre os mesmos é inadequado.

Alguns trabalhos realizados em nosso meio abordam o uso de medicamentos por pacientes idosos, como Huf et al. (2000) e Teixeira et al. (2000), entretanto, não explicitam o papel do farmacêutico na condução do regime terapêutico desse grupo de indivíduos.

Tendo em vista o apresentado, o objetivo deste trabalho foi fazer uma revisão da literatura para conhecer as publicações que relatam possíveis ações do farmacêutico, apresentando resultados quantitativos ou qualitativos, e como aquelas influenciam a farmacoterapia do paciente idoso.

\section{Materiais e métodos}

Buscou-se inicialmente trabalhos publicados no período de 1970 a outubro de 1999 em cinco bases de dados, com as seguintes estratégias de busca: (1) MEDLINE (descritores - Pharmacists/all subheadings and Aged/all subheadings and Prescriptions, Drug/all subheadings or Professional-Patient Relations/all subheadings or 
Physician-Patient Relations/all subheadings); (2) International Pharmaceutical Abstracts (IPA) (termo livre - Pharmacists and elderly or aged and Prescription Drugs or Prescribing Drugs); (3) Sociological Abstracts (descritores - Pharmacists in DE, Elderly in DE e Medications in DE); (4) PsycLIT (descritores - Pharmacists in DE, Aged in DE e Prescribing-Drugs in DE) e (5) Lilacs (descritores - farmacêutico, idoso e medicamento). Para a base Lilacs, em virtude do período de sua existência, o limite de tempo compreendido foi de 1981 a maio de 1999.

\section{Resultados}

Foram localizadas 55 publicações na base de dados MEDLINE. Na base IPA, 23 trabalhos foram encontrados. A busca localizou, ainda, cinco trabalhos na base Sociological Abstracts e oito publicações na base PsycLIT. Não se encontrou nenhuma publicação em relação aos descritores propostos na base Lilacs.

A maioria dos trabalhos referia-se à descrição de perfil de prescrição ou de problemas na utilização de medicamentos por idosos, outros relatavam alguma experiência ou intervenção mas não seu resultado. Esses artigos foram descartados da análise final, bem como outros que também não correspondiam aos objetivos do trabalho. Ou seja, não se referiam especificamente a idosos ou não permitiam inferir os resultados a esse grupo etário; referiam-se a medicamentos de venda livre e, ainda, os que se repetiam em outras bases de dados. Com isso, foram selecionadas 15 publicações, das 76 localizadas, para a análise final.

Não foi encontrado nenhum trabalho de intervenção publicado na década de 70 , tendo sido quatro artigos publicados na década de $80 \mathrm{e}$ 11 na de 90 . Dos 15 trabalhos analisados, 12 foram publicados nos Estados Unidos, um no Canadá, um no Reino Unido e um na Holanda, não tendo sido localizado nenhum artigo publicado em países de economia periférica.

A Tabela 1 apresenta as intervenções ocorridas no paciente idoso, sendo $67 \%$ (4/6) ações educativas e de aconselhamento ao paciente sobre seu regime terapêutico. Na Tabela 2, são apresentados os cinco trabalhos em que o farmacêutico atuou tanto no paciente idoso como no seu médico. Em quatro deles houve aconselhamento sobre o uso de medicamentos e, num outro, além dessas ações, o médico recebeu uma carta alertando sobre problemas com a prescrição. Todas as ações resultaram em melhoras no regime terapêutico ou no uso de medicamentos.
Como mostra a Tabela 3, três estudos referiam-se a intervenções exclusivamente nos médicos. As ações nos farmacêuticos, alocados em farmácias públicas, foram estudadas em um único trabalho.

Quando o objeto da ação foi o médico, a ação foi primordialmente de revisão da prescrição e de alerta ao prescritor. No caso da intervenção nos farmacêuticos, a proposta foi de educação continuada para capacitar o farmacêutico a detectar problemas com as prescrições de medicamentos de usuários de farmácias públicas.

\section{Discussão}

Os resultados apresentados permitem pressupor que, sob diferentes aspectos, os problemas relativos à medicação dos idosos têm sido mal equacionados. Há, além da flagrante falta de estudos, uma convergência quase sintomática de restrição da intervenção do farmacêutico ao processo de "capacitação ao uso" do medicamento ou das suas informações, como se esse profissional tivesse como única função "prover apoio" às decisões já tomadas de antemão. Essa redução implica ainda em maiores conseqüências, quando se considera as condições de economia periférica ou de transição, como é o caso brasileiro, onde os recursos humanos e materiais são escassos.

O número de publicações aumentou exponencialmente de uma década para outra, acompanhando a crescente relevância do idoso no perfil demográfico entre as economias avançadas, fonte de todas as publicações analisadas. Por outro lado, surpreende o reduzido número de estudos encontrados, dada a relevância do tema. É possível, porém, que as condições impostas para seleção dos trabalhos localizados tenham sido muito restritivas. Mesmo assim, o crescimento a partir da década de 90 foi expressivo. Nas décadas anteriores, os poucos trabalhos publicados tinham sua ênfase voltada para o conhecimento das particularidades do paciente idoso, bem como dos fatores que influenciam o uso de medicamentos nessa faixa etária. Foram apontados, entre outros, a falta de interação entre farmacêutico e paciente idoso, seus problemas cognitivos, psicológicos e as farmacoterapias complexas.

Os trabalhos da década de 90, por sua vez, enfatizam o aconselhamento ao paciente idoso e passam a valorizar mais a participação do farmacêutico e também do médico na melhoria da qualidade da prescrição médica. Surgem pesquisas sobre a redução do número de medicamentos prescritos, ajustes de dosagem, melho- 
Tabela 1

Estudos de intervenção do farmacêutico exclusivamente no paciente idoso, publicados no período de 1970 a maio de 1999.

\begin{tabular}{|c|c|c|}
\hline Tipo de intervenção & Objetivo da intervenção & Resultados da intervenção \\
\hline \multirow[t]{4}{*}{$\begin{array}{l}\text { Aconselhamentos sobre utilização } \\
\text { de medicamentos. }\end{array}$} & $\begin{array}{l}\text { Identificar problemas e descrever estratégias } \\
\text { para melhorar o comportamento relativo a } \\
\text { medicamentos (Hammarlund et al., } 1985 \text { - } \\
\text { Estados Unidos). }\end{array}$ & $\begin{array}{l}\text { Redução de } 11 \% \text { no número de prescrições. } \\
\text { Redução de } 39 \% \text { no número de problemas } \\
\text { de prescrição. }\end{array}$ \\
\hline & $\begin{array}{l}\text { Observar o efeito de ação educativa em } \\
\text { pacientes não institucionalizados com alto risco } \\
\text { de problemas com medicação (Sidel et al., } 1990 \text { - } \\
\text { Estados Unidos). }\end{array}$ & $\begin{array}{l}\text { Não houve mudança estatisticamente significante } \\
\text { entre os grupos estudado e controle sobre conhe- } \\
\text { cimento e práticas referentes a medicamentos. }\end{array}$ \\
\hline & $\begin{array}{l}\text { Testar um cartão recordatório de uso de } \\
\text { medicamentos em pacientes com regimes } \\
\text { medicamentosos complexos (Grymonpre } \\
\text { et al., } 1991 \text { - Canadá). }\end{array}$ & $\begin{array}{l}\text { Houve redução e interrupção de uso } \\
\text { de medicamentos de um paciente (1/12). } \\
\text { Útil para comunicar a outros profissionais } \\
\text { de saúde sobre o regime adotado. } \\
\text { Pacientes acharam fácil de ler e entender, }\end{array}$ \\
\hline & $\begin{array}{l}\text { Avaliar a intervenção do farmacêutico na } \\
\text { adesão ao tratamento com "colestipol" para } \\
\text { hipercolesterolemia (Konzem et al., } 1997 \text { - } \\
\text { Estados Unidos). }\end{array}$ & $\begin{array}{l}\text { mas acharam o formato grande e ruim. } \\
\text { Maior redução nas taxas de colesterol que no } \\
\text { grupo controle; maior adesão ao tratamento. }\end{array}$ \\
\hline $\begin{array}{l}\text { Farmacêuticos prescreveram } \\
\text { medicamentos (sob supervisão do } \\
\text { médico) e monitoraram seu uso } \\
\text { por pacientes institucionalizados. }\end{array}$ & $\begin{array}{l}\text { Determinar se a intervenção, na forma de } \\
\text { prescrição e administração de medicamentos, } \\
\text { poderia aumentar a qualidade no cuidado ao } \\
\text { paciente (Thompson et al., } 1984 \text { - Estados Unidos). }\end{array}$ & $\begin{array}{l}\text { Reduziram o custo reduzindo o número } \\
\text { de medicamentos. } \\
\text { Reduziram a taxa de hospitalização e } \\
\text { encaminharam pacientes a serviços com } \\
\text { menor complexibilidade, mais baratos. } \\
\text { Houve menos óbitos que no grupo controle. }\end{array}$ \\
\hline $\begin{array}{l}\text { Treinamento sobre auto- } \\
\text { administração de medicamentos a } \\
\text { pacientes em vias de alta hospitalar. }\end{array}$ & $\begin{array}{l}\text { Treinar os pacientes para que pudessem auto- } \\
\text { administrar-se medicamentos em casa, após a alta } \\
\text { hospitalar (Walker \& Martin, } 1986 \text { - Reino Unido). }\end{array}$ & $\begin{array}{l}70 \% \text { dos pacientes ficaram totalmente } \\
\text { independentes para auto-administrar-se } \\
\text { medicamentos quando foram para casa. } \\
\text { Redução de encargos dos cuidadores. }\end{array}$ \\
\hline
\end{tabular}

Tabela 2

Estudos de intervenção do farmacêutico no paciente idoso e seu médico prescritor, publicados no período de 1970 a maio de 1999 .

\begin{tabular}{|c|c|c|}
\hline Tipo de intervenção & Objetivo da intervenção & Resultados da intervenção \\
\hline \multirow[t]{4}{*}{$\begin{array}{l}\text { Aconselhamento ao médico } \\
\text { e ao paciente. }\end{array}$} & $\begin{array}{l}\text { Avaliar um serviço pago de acompanhamento por } \\
\text { farmacêuticos (Gehres, } 1986 \text { - Estados Unidos). }\end{array}$ & $\begin{array}{l}\text { Houve alterações no regime terapêutico. } \\
\text { Houve aumento de adesão. }\end{array}$ \\
\hline & $\begin{array}{l}\text { Testar consultas de farmacêuticos clínicos como } \\
\text { forma de melhorar a prescrição médica a pacientes } \\
\text { ambulatoriais (Lipton et al., } 1992 \text { - Estados Unidos). }\end{array}$ & $\begin{array}{l}\text { Redução do número de problemas com as } \\
\text { prescrições (alergias, dosagem, interações). }\end{array}$ \\
\hline & $\begin{array}{l}\text { Avaliar o impacto da intervenção do farmacêutico } \\
\text { clínico em adesão, redução de problemas com } \\
\text { prescrição e readmissões hospitalares (Lipton et } \\
\text { al., } 1994 \text { - Estados Unidos). }\end{array}$ & $\begin{array}{l}\text { Aumento de adesão e maior conhecimento do } \\
\text { paciente sobre seu regime terapêutico. }\end{array}$ \\
\hline & $\begin{array}{l}\text { Estimar o custo da intervenção de um farmacêutico } \\
\text { clínico na prescrição de pacientes não institucionali- } \\
\text { zados, do ponto de vista do sistema de saúde } \\
\text { (Cowper et al., } 1998 \text { - Estados Unidos). }\end{array}$ & $\begin{array}{l}\text { Não houve redução dos custos diretos mas houve } \\
\text { redução nos problemas de prescrição. }\end{array}$ \\
\hline $\begin{array}{l}\text { Carta para o médico após } \\
\text { avaliação do histórico de } \\
\text { medicamentos do paciente }+ \\
\text { entrevistas com o paciente. }\end{array}$ & $\begin{array}{l}\text { Avaliar se a intervenção do farmacêutico em } \\
\text { pacientes não institucionalizados reduziria problemas } \\
\text { de prescrição (Grymonpre et al., } 1994 \text { - Canadá). }\end{array}$ & $\begin{array}{l}\text { Mudanças na medicação consistentes com } \\
\text { as recomendações. }\end{array}$ \\
\hline
\end{tabular}


Estudos de intervenção do farmacêutico sobre profissionais de saúde envolvidos na medicação de idosos, publicados no período de 1970 a maio de 1999.

\begin{tabular}{|c|c|c|}
\hline $\begin{array}{l}\text { Sujeito da intervenção/ } \\
\text { Tipo de intervenção }\end{array}$ & Objetivo da intervenção & Resultados \\
\hline \multicolumn{3}{|l|}{ Farmacêutico } \\
\hline $\begin{array}{l}\text { Treinamento de farmacêuticos } \\
\text { (manual + workshop). }\end{array}$ & $\begin{array}{l}\text { Educação de farmacêuticos de farmácias públicas } \\
\text { para detectar problemas relacionados a medica- } \\
\text { mentos (Kimberlin et al., } 1993 \text { - Estados Unidos). }\end{array}$ & $\begin{array}{l}\text { Os farmacêuticos treinados ficaram mais } \\
\text { engajados nas atividades de educação } \\
\text { de seus pacientes. } \\
\text { O efeito nos pacientes perdurou até três } \\
\text { meses após a intervenção. }\end{array}$ \\
\hline \multicolumn{3}{|l|}{ Médico } \\
\hline $\begin{array}{l}\text { Revisão das prescrições dos } \\
\text { pacientes institucionalizados. }\end{array}$ & $\begin{array}{l}\text { Observar o efeito da ação de um farmacêutico } \\
\text { clínico geriatra na prescrição de medicamentos a } \\
\text { idosos (Phillips \& Carr-Lopez, } 1990 \text { - Estados Unidos). }\end{array}$ & $\begin{array}{l}\text { Redução de } 32 \% \text { no número de prescrições } \\
\text { e de } 42 \% \text { no número de medicamentos } \\
\text { associados a reações adversas. } \\
\text { Redução nos custos diretos por paciente. }\end{array}$ \\
\hline $\begin{array}{l}\text { Chamadas telefônicas ao médico } \\
\text { quando se observavam problemas } \\
\text { na prescrição. }\end{array}$ & $\begin{array}{l}\text { Avaliar a utilidade de um banco de dados on line, } \\
\text { capaz de detectar uso inapropriado de medicamentos } \\
\text { por usuários do serviço postal de farmácia (Monane } \\
\text { et al., } 1998 \text { - Estados Unidos). }\end{array}$ & $\begin{array}{l}\text { Em média, houve } 24 \% \text { de mudanças para } \\
\text { um agente terapêutico mais indicado. }\end{array}$ \\
\hline $\begin{array}{l}\text { Recomendações ao prescritor sobre } \\
\text { os medicamentos usados por seu } \\
\text { paciente. }\end{array}$ & $\begin{array}{l}\text { Investigar se e como a ação do farmacêutico } \\
\text { influenciaria a prescrição de medicamentos } \\
\text { por clínicos gerais (Strikwerda et al., } 1994 \text { - Holanda). }\end{array}$ & $\begin{array}{l}\text { Redução de } 7 \% \text { no número de prescrições. } \\
\text { Redução de dosagem em quatro pacientes. } \\
\text { Interrupção de uso de medicamentos em } \\
\text { nove pacientes. }\end{array}$ \\
\hline
\end{tabular}

ra na seleção de medicamentos e redução do número de reações adversas, além do uso de bancos eletrônicos de informações sobre medicamentos. Em nenhum dos estudos o objeto da pesquisa foi o medicamento em si.

Com essa primazia concedida aos aconselhamentos, tanto ao paciente como ao médico, os farmacêuticos vêm buscando, sob diferentes formas, reduzir custos ou aumentar a efetividade dos tratamentos, a partir de intervenções na forma como o idoso, o médico prescritor ou mesmo os próprios farmacêuticos lidam com o medicamento ou com as suas informações. Nesse processo, o desfecho nem sempre foi plenamente favorável ou isento de conflitos. Cowper et al. (1998), por exemplo, buscavam estimar o custo da intervenção de um farmacêutico clínico na adequação da prescrição de medicamentos. Não houve redução nos custos diretos, conforme esperado pelos autores, uma vez que a intervenção priorizava a otimização do regime terapêutico. Segundo eles, foi observada uma adequação dos regimes na forma de redução de alguns problemas com a prescrição, como a subdosagem. Em contrapartida, os custos não puderam ser reduzidos, possivelmente pelo aumento do número de medicamentos prescritos ou pelo maior custo dos remédios introduzidos em substituição aos problemáticos. Também Sidel et al. (1990) não veri- ficaram diferenças estatisticamente significantes entre um grupo de pacientes que submeteu-se a orientações sobre medicamentos e um grupo controle. Além de possíveis viéses, os farmacêuticos alegaram dificuldades para obtenção de "mudança de atitudes" sobre medicamentos na população de estudo. A alegada resistência dos idosos em mudar os seus hábitos e práticas passa a justificar outras propostas, como a ação educativa continuada de Hammarlund et al. (1985), cujo "êxito" em reduzir significativamente o número de medicamentos usados foi atribuído a esse gênero de intervenção.

Os estudos analisados reforçam a idéia de que a intervenção do farmacêutico aumenta a adesão dos pacientes idosos a seus regimes terapêuticos, e pode promover redução de custos hospitalares, ao reduzir o número de prescrições, de internações e de medicamentos associados a RAMs (Grymonpre et al., 1994; Phillips \& Carr-Lopez, 1990; Thompson et al., 1984; Walker \& Martin, 1986). Em particular, Gehres (1986) relata a experiência de um serviço pago de acompanhamento de farmacêuticos a idosos, cuja atenção permitiu a auto-administração de medicamentos e a possibilidade de vida junto a instituições de apoio à saúde de menor complexidade e, portanto, mais baratas. Entretanto, sob o ponto de vista do processo, há uma recorrente dependência da viabilização de "for- 
mas comunicativas”. Lipton \& Bird (1994) apontam que a primeira entrevista que tiveram com pacientes após a alta hospitalar, mostrou-se principalmente de sensibilização. Apenas três meses após o início do monitoramento e aconselhamento foi observado impacto no uso. Segundo os autores, a razão para essa demora pode ter sido a necessidade de reforço contínuo para aumentar a adesão.

Não é sem razão, portanto, a ênfase em se minimizar barreiras na comunicação farmacêutico-paciente idoso. Espera-se que, com a melhoria desse procedimento, os pacientes administrem melhor os riscos associados com o uso dos medicamentos (Kessler, 1991; Wiederholt et al., 1992). Nesse sentido, Pepe \& Castro (2000) ressaltam que a informação prestada ao paciente no ato da dispensação é tão ou mais importante que o medicamento por ele recebido. Com o enfoque centrado dessa forma, não é sem razão também que os resultados sejam por vezes efêmeros, exigindo a ação continuada.

As ações do farmacêutico sobre o prescritor foram semelhantes e os resultados também não foram diferentes. Grymonpre et al. (1994) revelaram que ao avaliar prescrições de pacientes não institucionalizados, encaminhando alertas sobre inadequações da prescrição a seus médicos, apenas $31,3 \%$ desses alertas resultaram em mudanças. Questionados sobre a utilidade daquele serviço, 81,8\% dos médicos classificaram como útil ou muito útil, mas os demais o consideraram "perda de tempo". Os demais estudos mostram que a rejeição do apoio do farmacêutico, ou "resistência" do médico, cai quando as ações são conjuntas, quando ambos faziam parte do mesmo projeto ou da mesma equipe de saúde. Dessa forma, os médicos podem avaliar a ação do farmacêutico como uma ingerência menos invasiva em sua atividade profissional. Isso faz com que a proposta de uso de bancos de dados on-line, com geração de alertas sobre problemas na prescrição, venha a constituir uma interface mais produtiva entre médico-farmacêutico, dado os custos de manutenção de uma equipe multiprofissional.

A prescrição inadequada aos idosos é freqüentemente atribuída à falta de treinamento de prescritores em geriatria e na deficiência da formação farmacêutica. Embora os recursos modernos de acesso à informação possam resolver parte desse problema, como propõem Monane et al. (1998), é fato que o idoso vive um contexto próprio em que alguns aspectos podem ser generalizados e outros não. No processo que envolve a terapia farmacológica, o farmacêutico, por ocasião da dispensação do medicamento, mantém contato com paciente ido- so e tem uma oportunidade concreta de avaliar se ele possui incapacidades que possam comprometer o seu acesso ou a sua adesão ao tratamento medicamentoso (Thwaites, 1999). Trata-se, em essência, de uma forma diferente de se entender a prática profissional, onde o foco passa a ser o cuidado com o paciente (Hepler \& Strand, 1990). Embora os programas educativos instituídos pelo farmacêutico possam mostrarse efetivos para garantir independência e utilização adequada de medicamentos, principalmente reduzindo erros de prescrições ou de dosagem, prevenindo uso incorreto e reações adversas (Walker \& Martin, 1986), há de se ter em conta que o farmacêutico dispõe de capacitação técnica para promover muitas outras adequações. Os idosos compartilham problemas como dificuldades visuais, de memória, força muscular e outras que obrigam, por exemplo, a revisão das formas farmacêuticas, das embalagens e dos rótulos entre outros. São poucos, como Galizia \& Sause (1982), que chamam a atenção para esses aspectos mais gerais. Além desses, os idosos encontram-se, freqüentemente, também sujeitos a problemas de ordem pessoal, como aqueles decorrentes da auto-estima, da solidão ou da marginalização circunstancial. Ranelli \& Coward (1997), por exemplo, verificaram que idosos moradores de zona rural tinham expectativas diferentes dos residentes em zona urbana quanto à atenção do farmacêutico.

A proposição de medidas deve, portanto, levar em conta esse conjunto maior, que constitui o universo de exigências circunstanciais do idoso. Só assim pode-se entender a "resistência" como uma reação natural às necessidades não atendidas, como exemplifica bem a rejeição ao "cartão de memória” pelos pacientes, embora fosse muito conveniente aos profissionais de saúde, conforme relatado por Grymonpre et al. (1991). Como lembram Caprara \& Franco (1999), ao discutir a humanização da relação médico-paciente, os profissionais de saúde devem ter sensibilidade para conhecer a realidade do paciente e ouvir suas queixas. E nesses termos, também cabe questionar o quanto dessas experiências apresentadas é conveniente à condição brasileira.

Kimberlin et al. (1993) afirmaram que os farmacêuticos, alocados em farmácias públicas dos Estados Unidos, apresentavam condições de assumir responsabilidade e de propor resolução de problemas relacionados a medicamentos relatados por pacientes não institucionalizados. Já Jones et al. (1997) relataram que $79 \%$ dos pesquisados em sua investigação no País de Gales nunca discutiriam informações 
sobre medicamentos com os farmacêuticos, preferindo fazê-lo com seu próprio médico. No Brasil, onde o sistema de saúde por vezes não consegue atender a demanda de usuários, o atendente na farmácia pública, será, com freqüência, o único profissional com o qual o paciente irá relacionar-se. Além disso, o tempo despendido para a atenção farmacêutica também deve ser considerado nas avaliações de custo. No caso do cartão recordatório proposto por Grymonpre et al. (1991) demorava-se uma média de 25 minutos para completar o cartão e outros 20 minutos para aconselhar o paciente. Walker \& Martin (1986) relataram que a média de tempo dispensado a cada paciente era de 50 a 60 minutos. A possibilidade de intervenções desse gênero no Brasil precisa levar em conta as condições específicas, em que ainda há escassez de farmacêuticos atuando em farmácia clínica, como também na atenção primária no sistema de saúde.

Tal como observado por Galizia \& Sauce (1982) nos Estados Unidos, também no Brasil pode-se esperar problemas relativos à linguagem, à alfabetização deficiente ou mesmo às formas de entendimento, os quais podem ser resolvidos com medidas específicas, como propõem esses autores. A solução desses e de outros problemas implica num esforço imaginativo por parte do profissional de saúde, assim como da implementação de tecnologias atuais. Já há no Brasil, por exemplo, programas de computador para serem utilizados em farmácias públicas (PAC System, 2002), bem como em hospitais, com capacidade de gerar alertas sobre a medicação e permitir acompanhamento do paciente. Além disso, o emprego de telefone para seguimento de pacientes, como usado por Ranelli \& Coward (1997), não deve ser descartado. Em nosso meio, a expansão da telefonia, fixa e móvel, foi expressiva nos últimos anos, poden- do ser uma alternativa de acompanhamento, especialmente nas áreas urbanas.

\section{Conclusão}

A revisão da literatura, descrevendo e qualificando a intervenção de farmacêuticos na farmacoterapia do paciente idoso, mostrou que estudos sobre o tema são escassos e limitados aos países de economia avançada. De uma forma geral, as intervenções mostraram resultados positivos, reduzindo custos, melhorando as prescrições, promovendo maior adesão do paciente ao tratamento e controlando a possibilidade de reações adversas. Ao mesmo tempo, os trabalhos revistos mostraram que a natureza das intervenções fica ainda muito restrita às diferentes possibilidades de aconselhamento do usuário e do prescritor de medicamentos. Essa falta de posicionamento mais pró-ativo do profissional farmacêutico, buscando também a adequação do medicamento ao usuário, reduz o seu papel no sistema de saúde e compromete o atendimento das necessidades de países como o Brasil.

Em vista do encontrado, se fazem necessárias diferentes investigações específicas. Pouco se sabe, por exemplo, sobre as necessidades que caracterizam o idoso brasileiro nos diferentes contextos sociais em que ele se encontra, bem como sobre as formas de solução possíveis para atendê-las. Não se encontram descrições disponíveis sobre as experiências bem ou mal sucedidas na farmacoterapia do idoso em ambiente hospitalar ou doméstico brasileiro. Além disso, faltam investigações que mostrem as possibilidades da ciência farmacêutica, nas suas diferentes áreas de conhecimento, em atender os problemas particulares desse grupo etário. 


\section{Referências}

ATKIN, P. A. \& SHENFIELD, G. M., 1995. Medication related adverse reactions and the elderly: A literature review. Adverse Drug Reaction Toxicology Review, 14:175-191.

BAUM, C.; KENNEDY, D. L. \& FORBES, J. K., 1984. Drug use in the United States in 1981. JAMA, 251:12931297.

CAPRARA, A. \& FRANCO, A. L. S., 1999. A relação médico-paciente: Para uma humanização da prática médica. Cadernos de Saúde Pública, 15:647-654.

CARTWRIGHT, A. \& SMITH, C., 1988. Elderly People, Their Medicines and Their Doctor. New York: Routledge.

COWPER, P. A.; WEINBERGER, M.; HANLON, J. T.; LANDSMAN, P. B.; SAMSA, G. P.; UTTECH, K. M.; SCHMADER, K. E.; LEWIS, I. K.; COHEN, H. J. \& FEUSSNER, J. R., 1998. The cost-effectiveness of a clinical pharmacist intervention among elderly outpatients. Pharmacotherapy, 18:327-332.

DHSS (U.S. Department of Health and Human Services), 2000. Administration on Aging-Older Americans Month 2001. The Many Faces of Aging. December $2000<$ http://www.os.dhhs.gov/topics/ aging.htlm>.

GALIZIA, V. J. \& SAUSE, R. B., 1982. Communicating with the geriatric patient. American Pharmacy, NS22:35-36.

GEHRES, R. W., 1986. Medication monitoring services for elderly patients offered by the pharmacist on a fee for service basis. Journal of Geriatric Drug Therapy, 1:81-89.

GIBBS, S.; WATERS, W. E. \& GEORGE, C. F., 1989. The benefits of prescription information leaflets. British Journal of Clinical Pharmacology, 27:723739.

GRYMONPRE, R.; SABISTON, C. \& JOHNS, B., 1991. Development of a medication reminder card for elderly persons. Canadian Journal of Hospital Pharmacy, 44:55-62.

GRYMONPRE, R. E.; WILLIAMSON, D. A.; HUYNH, D. H. \& DESILETS, L. M., 1994. Community-based pharmaceutical care model for the elderly: Report on a pilot project. International Journal of Pharmacy Practice, 2:229-234.

HAMMARLUND, E. R.; OSTRORN, J. R. \& KETHLEY, A. J., 1985. The effects of drug counseling and other educational strategies on drug utilization of the elderly. Medical Care, 23:165-170.

HELLER, T. A.; LARSON, E. B. \& LoGERFO, J. P., 1984. Quality of ambulatory care of the elderly: An analysis of five conditions. Journal of the American Geriatrics Society, 32:782-788.

HEPLER, C. D. \& STRAND, L. M., 1990. Opportunities and responsibilities in pharmaceutical care. American Journal of Hospital Pharmacy, 47:533-543.

HUF, G.; LOPES, C. S. R. \& ROZENFELD, S., 2000. O uso prolongado de benzodiazepínicos em mulheres de um centro de convivência para idosos. Cadernos de Saúde Pública, 16:351-362.

IBGE (Fundação Instituto Brasileiro de Geografia e Estatística), 1998. Anuário Estatístico do Brasil: 1998. Rio de Janeiro: IBGE.

IBGE (Fundação Instituto Brasileiro de Geografia e Estatística), 1999. Pesquisa Nacional por Amostra de
Domicílio - PNAD. População Residente, Total e por Sexo, Segundo os Grupos de Idade - 1999 - Brasil. Março 2001 <http://www.ibge.gov.br/home/ estatistica/populacao/trabalhoerendimento/ pnad99/sintese/images/tabeladegraficolb.shtm>.

JONES, D.; SEYMOUR, R. \& WOODHOUSE, K., 1997. Use of pharmacists by older people in the community. Archives of Gerontology and Geriatrics, 24:9-13.

KESSLER, D. A., 1991. Communicating with patients about their medications. New England Journal of Medicine, 325:1650-1652.

KESSLER, D. A., 1992. The FDA commissioner challenges pharmacists to renew their commitment to patient education. American Pharmacy, 32:33-36.

KIMBERLIN, C. L.; BERARDO, D. H.; PENDERGAST, J. F. \& McKENZIE, L. C., 1993. Effects of an education program for community pharmacists on detecting drug-related problems in elderly patients. Med ical Care, 31:451-468.

KONZEM, S. L.; GRAY, D. R. \& KASHYAP, M. L., 1997. Effect of pharmaceutical care on optimum colestipol treatment in elderly hypercholesterolemic veterans. Pharmacotherapy, 17:576-583.

LAMY, P., 1990. Adverse drug effects. Clinics in Geriatric Medicine, 6:293-307.

LANDAHL, S., 1987. Drug treatment in 70-82-year-old persons. A longitudinal study. Acta Medica Scandinavica, 221:179-184.

LIPTON, H. L.; BERO, L. A.; BIRD, J. A. \& McPHEE, S. J., 1992. The impact of clinical pharmacists' consultations on physicians' geriatric drug prescribing: A randomized controlled trial. Medical Care, 30:646-655.

LIPTON, H. L. \& BIRD, J. A., 1994. The impact of clinical pharmacists' consultations on geriatric patients' compliance and medical care use: A randomized controlled trial. Gerontologist, 34:307-315.

MONANE, M.; MATTHIAS, D. M.; NAGLE, B. A. \& KELLY, M. A., 1998. Improving prescribing patterns for the elderly through an online drug utilization review intervention: A system linking the physician, pharmacist, and computer. JAMA, 280: 1249-1252.

MORROW, D.; LEIRER, V. \& SHEIKH, J., 1988. Adherence and medication instructions: Review and recommendations. Journal of the American Geriatrics Society, 36:1147-1160.

MS (Ministério da Saúde), 1999. Programa de Saúde do Idoso. Março 2001 <http://www.saude.gov.br/ programas/idoso/programa.htm>.

NOLAN, L. \& O'MALLEY, K., 1988. Prescribing for the elderly: Part II. Prescribing patterns: Differences due to age. Journal of the American Geriatrics Society, 36:245-254.

O'CONNELL, M. B. \& JOHNSON, J. F., 1992. Evaluation of medication knowledge in elderly patients. $A n$ nals of Pharmacotherapy, 26:919-921.

OKUNO, J.; YANAGI, H.; TOMURA, S.; OKA, M.; HARA, S.; HIRANO, C. \& TSUCHIYA, S., 1999. Compliance and medication knowledge among elderly Japanese home-care recipients. European Journal of Clinical Pharmacology, 55:145-149.

ÖSTERLIND, P. \& BUCHT, G., 1991. Drug consumption 
during the last decade among persons born in 1902 in Umeä, Sweden. A longitudinal population study. Drugs \& Aging, 1:477-486.

OSTROM, J. R.; HAMMARLUND, E. R.; CHRISTENSEN, D. B.; PLEIN, J. B. \& KETHLEY, A. J., 1985. Medication usage in an elderly population. Medical Care, 23:157-164.

PAC SYSTEM, 2002. Programa de Assistência ao Consumidor. CD-ROM. São Paulo: Grupo Zanini-Oga/ Altemate.

PEPE, V. L. E. \& CASTRO, C. G. S. O., 2000. A interação entre prescritores, dispensadores e pacientes: Informação compartilhada como possível beneficio terapêutico. Cadernos de Saúde Pública, 16: 815-822.

PHILLIPS, S. L. \& CARR-LOPEZ, S. M., 1990. Impact of a pharmacist on medications discontinuation in a hospital-based geriatric clinic. American Journal of Hospital Pharmacy, 47:1075-1079.

PIRAINO, A. J., 1995. Managing medication in the elderly. Hospital Practice, 30:59-64.

RANELLI, P. L. \& COWARD, R. T., 1997. Communication between pharmacists and patients: The role of place of residence in determining the expectations of older adults. Pharmacotherapy, 17:148-162.

SALOM, I. L. \& DAVIS, K., 1995. Prescribing for older patients: How to avoid toxic drug reactions. Geriatrics, 50:37-40,43-45.

SIDEL, V. W.; BEIZER, J. L.; LISI-FAZIO, D.; KLEINMANN, K.; WENSTON, J.; THOMAS, C. \& KELMAN, H. R., 1990. Controlled study of the impact of educational home visits by pharmacists to high-risk older patients. Journal of Community Health, 15: 163-174.
STRIKWERDA, P.; BOOTSMA-DE LANGEN, A. M.; BERGHUIS, F. \& MEYBOOM-DE JONG, B., 1994. Farmacotherapie in een verzogingshuis; Gunstinge invloed van feed-back door de apotheker op het voorschrijfgedrag van de huisarts. Nederlands Tijdschrift voor Geneeskunde, 138:1770-1774.

SULLIVAN, S. D.; KRELING, D. H. \& HAZLET, T. K., 1990. Noncompliance with medication regimens and subsequent hospitalizations: A literature analysis and cost of hospitalization estimate. Journal of the Research in Pharmaceutical Economics, 2:19-33.

TEIXEIRA, J. J. V.; LEFEVRE, F.; CASTRO, L. L. C. \& SPÍNOLA, A. W. P., 2000. Drug compliance and the elderly: Who is publishing, where and when? $\mathrm{Ca}$ dernos de Saúde Pública, 16:139-144.

THOMPSON, J. F.; McGHAN, W. F.; RUFFALO, R. L.; COHEN, D. A.; ADAMCIK, B. \& SEGAL, J. L., 1984. Clinical pharmacists prescribing drug therapy in a geriatric setting: Outcome of a trial. Journal of the American Geriatric Society, 32:154-159.

THWAITES, J. H., 1999. Practical aspects of drug treatment in elderly patients with mobility problems. Drugs \& Aging, 14:105-114.

WALKER, C. \& MARTIN, P. C., 1986. Inpatient self-medication in the elderly-pilot scheme. Pharmacy Journal, 237:767-768.

WALKER, J. \& WYNNE, H., 1994. Review: The frequency and severity of adverse drug in elderly people. Age and Ageing, 23:255-259.

WIEDERHOLT, J. B.; CLARRIDGE, B. R. \& SVARSTAD, B. L., 1992. Verbal consultation regarding prescription drugs: Findings from a statewide study. Medical Care, 30:159-173.

Recebido em 17 de maio de 2001

Versão final reapresentada em 13 de março de 2002

Aprovado em 4 de julho de 2002 